\title{
From experimental procedure to clinical practice: how much evidence do we need in Uterine Transplantation?
}

Sabina Gainotti, Bioethics Unit, Office of the President, Istituto Superiore di Sanità

V.le Regina Elena, 299 CAP 00162, Rome, Italy

sabina.gainotti@iss.it

Carlo Petrini, Head of the Bioethics Unit, Office of the President, Istituto Superiore di Sanità

V.le Regina Elena, 299 CAP 00162, Rome, Italy

carlo.petrini@iss.it

Corresponding author: Sabina Gainotti

\section{Abstract}

\section{Background}

Absolute uterine factor infertility (AUFI) is a kind of infertility that is completely attributable to uterine absence (surgical or congenital for women with MayerRokitansky-Küster-Hauser syndrome: MRKH) or anatomic or functional abnormality that prevents embryo implantation or completion of pregnancy to term.

Until recently, the only viable option to parenthood for couples with AUFI were adoption or surrogacy.

Since a first attempt of uterus transplant (UTx) in 2000, nine babies were born from women with a transplanted uterus from 2014, eight of which in Sweden, and one in the United States.

These promising results are raising immense hopes for the women with AUFI and there is optimism about the possibility for UTx to become part of clinical care even though, besides encouraging results, the procedure has also resulted in increased risks and harms for both the donors and recipients and increased risks of premature birth for the fetus.

At present UTx is still considered as experimental and requiring more research and safety assessment before becoming a therapeutic option for AUFI. The transition from experimental procedure to therapeutic care would result in less strict ethical scrutiny for UTx and in the possibility for patients to get 
reimbursement for the procedure by the relevant healthcare insurance or public healthcare providers. In turn, an increase in the number of UTx performed yearly by specialized surgical teams would result in a general improvement of the "field strength".

However, at present it is difficult to establish the amount of evidence that we need in order to consider UTx as no longer experimental but routine clinical practice.

The literature on UTx provides recommendations on the different outcomes that should be monitored in this experimental phase but no study is anticipating the number of subjects that should be followed and for how long.

\section{Conclusion}

As for other transplants that have become routine practice, like renal transplant and heart transplant, it is likely that the decision on "routine practice readiness" will result from available cumulated evidences, from expert capacity to find a consensus on best practices and on political considerations as well, including pressures form patients and patient groups.

\section{Plain English summary}

Progresses in Assisted Reproduction Technologies (ART) are overcoming most causes of infertility in women and men, like ovulation problems and tubal blockage, low sperm counts, and poor sperm quality.

Absolute uterine factor infertility (AUFI) is a kind of infertility that is completely attributable to the absence or malfunctioning of the uterus, which can be congenital in women with Mayer-Rokitansky-Küster-Hauser syndrome (MRKH) or it can be acquired following surgery (hysterectomy).

Until recently, the only viable option to parenthood for couples with AUFI were adoption or surrogacy.

However, several surgical equips are performing uterus transplants (UTx) worldwide and, since 2014, 9 babies were born from women with a transplanted uterus, eight of which in Sweden and one in the United States.

UTx is still considered as an experimental transplant, requiring more research and safety assessment before becoming a therapeutic option for AUFI.

There is optimism about the possibility that it become part of clinical care even though, besides encouraging results, UTx is also resulting in increased risks and harms for the donors and recipients and increased risks of premature birth for the fetus.

There is no clarity on the number of interventions that need to be performed before considering UTx as routine clinical practice. 
As for other transplants, like renal transplant and heart transplant, it is likely that the decision on "routine practice readiness" will depend on the results of current interventions, from expert capacity agree on best practices and on political considerations as well, including pressures form patients groups.

\section{Keywords}

Absolute Uterine Factor Infertility, Uterus Transplant, Experimental, Clinical Practice, Ethics, Living Donors, Risk Assessment

\section{List of abbreviations}

Absolute Uterine Factor Infertility (AUFI)

Assisted Reproduction Technologies (ART)

Federation of Gynecology and Obstetrics (FIGO)

Hospital Anxiety and Depression Scale (HADS)

Idea; Development; Exploration; Assessment; Long-term study (IDEAL)

Independent Data Monitoring Committee (IDMC)

In Vitro fertilization (IVF)

Mayer-Rokitansky-Küster-Hauser syndrome (MRKH)

Uterine Transplantation (UTx)

World Medical Association (WMA)

\section{Background}

Like all clinical advances, UTx involves new problems of uncertainty concerning the state of development of the procedure, and how much experimental or therapeutic it should be considered.

It has been proposed that all medical treatment is in some way "experimental" due to the many levels of uncertainty in medical practice, deriving from the limitations in current medical understanding and techniques, the physician's incomplete 
mastery of available knowledge and skills, and the difficulty in distinguishing between the two previous uncertainties [1].

Even so, the question if a medical procedure is to be considered experimental or routine practice has huge practical implications, in particular for deciding on whom and under what circumstances it may be justifiably used and whether its costs will be reimbursed by the health insurance or public healthcare coverage $[2,3]$.

In pharmacological research, the transition from experimental procedure to clinical practice is usually sanctioned by the formal act of Marketing Authorization. In the EU pharmaceutical companies must submit a single marketing-authorisation application to European Medicine Agency (EMA) where they present the results of pharmaceutical (physico-chemical, biological or microbiological) tests, pre-clinical (toxicological and pharmacological) tests, and clinical trials [4].

Similarly, in the US pharmaceutical companies must submit a New Drug Application (NDA) to the Food and Drug administration (FDA) supported by the data gathered during animal studies and human clinical trials of an Investigational New Drug (IND) before the new pharmaceutical is approved by the FDA for sale and marketing [5]. In transplant surgery (TS) the transition from experimental procedure to normal care is less clear-cut and the procedure for the assessment of the procedure is not centralized at the EU or US federal level.

This may be due to a difficulty to "control" the determinants of relevant health outcomes in TS, which depend much on operator, team, and setting, such as learning curves, quality variations, and perception of equipoise [6].

Frequently, in surgery, new procedures are the result of small step-by-step departures from protocols already established in clinical practice; or they may be 
the result of radical innovation. If the new techniques are successful, they may become the basis of new protocols [7].

Criteria for ethical analysis of surgical innovation were proposed by Moore in 2000 and include laboratory background, field strength, and institutional stability [8]. Laboratory background requires that any research effort be made in several animal species and follow a step-by-step procedure, in order to optimize the intervention and to increase safety before trying the procedure with the human. Field strength requires that adequate synthesis of knowledge and expertise is available from all fields related to the procedure. Institutional stability addresses the overall level of expertise in the institution in which the procedure is performed, including the quality of all clinical services, support services and their capacity to interact in an interdisciplinary manner [9].

More recently, McCulloch et al proposed the IDEAL recommendations for the assessment of surgery based on a five-stage description of the surgical development process, including: 1) Idea; 2a) Development; 2b) Exploration; 3) Assessment; 4) Long-term study [10].

The four IDEAL phases partly correspond to the four phases of clinical trials, in that their main purposes and outcomes go from the proof of concept in stage 1, to the focus on safety in stage $2 a$ and stage $2 b$, to a deeper evaluation of clinical outcomes in stage 3 to surveillance and assessment of long term outcomes in stage 4.

However, as compared to pharmacological research, surgery often lacks centralized regulatory authorities that require studies of efficacy before a new procedure can be offered to the patients [11]. 
The decision about "routine practice readiness" of a surgical procedure thus seems to rely on expert capacity to find a consensus based on best practices (i.e. Moore's criteria and the IDEAL recommendations) and on ad hoc criteria as well.

As compared to the standardized procedure for drugs approval, in TS the political dimension also seems to play a relevant role even if it is not required by predefined procedures [12].

\section{Benefits and risks of UTx for the recipient, the donor and the future} child

Since the first attempt in 2000 up today uterine transplantation (UTx) has been documented in several women with absolute uterine factor infertility (AUFI), a kind of infertility that is completely attributable to uterine absence (surgical or congenital for women with Mayer-Rokitansky-Küster-Hauser syndrome: MRKH) or anatomic or functional abnormality that prevents embryo implantation or completion of pregnancy to term.

The first interventions were performed in 2000 in Saudi Arabia and in 2011 in Turkey. The first failed as the uterus had to be removed after two weeks, the second was more successful as after more than 5 years the uterus was still in place, but the transplant was followed by two consecutive pregnancies and abortions [14, 15]. In the Swedish UTx trial, nine live-donor UTx procedures were performed at Gothenburg in early 2013. Two uterus had to be removed a few weeks after the surgery, due to infection or circulatory problems, while seven out of the nine transplanted uteri were still in place after 6-months [16], and a total of 6 livebirth have been documented after the procedure, with one patient giving birth twice 
[17]. Two more pregnancies were ongoing in 2016 and expected to deliver in early summer 2017 [15].

At the Baylor University Medical Center at Dallas, US, a total of $8 / 10$ uterus transplants have been completed, using a living donor uterus, three of which have failed. Of the remaining five, one has resulted in a livebirth at the end of 2017 and the hospital has confirmed that there is another woman in the trial who is pregnant. [18].

It must be noted that, as compared to other transplants, success rate in UTx is only partially determined by successful operations, as the final outcome here is the birth of a healthy child, which is depending on a huge number of factors.

As regarding safety, the risks and benefits to be assessed in the short and in the long run are multiple in UTx and they concern:

- $\quad$ The safety and efficacy for the recipient;

- $\quad$ The safety for the donor if living donation is considered as an option;

- The safety for the child and in particular the risk of premature birth and its consequences.

For the recipient, the possibility of suffering harms from the procedure are potentially compensated by the possibility of achieving the desired outcome, that is experiencing pregnancy and hopefully giving birth to a healthy child, and by the possibility to autonomously express this willingness throughout the informed consent process.

There are women with AUFI who have a strong emotional and social desire to carry a pregnancy and, if correctly informed, they alone, even supported and counseled 
by their partner and the transplant team, can decide to undergo such a difficult and risky path.

If it achieves the intended outcome, UTx may result in health benefits for the patient and the couple.

The experience of pregnancy and parenthood are likely to result in reduced psychological stress and a general improvement of the woman's well-being and quality of life, which might also be shared by her partner, and these effects may remain in the long term [19].

However, there are many known and unknown physical and psychological risks in UTx related to surgery, immunosuppression, pregnancy, and childbirth.

The surgical risks include infections (mostly urinary tract and wound infections), perioperative bleeding, and thrombosis. General anaesthesia also carries a risk for the patient even though the risk of mortality is very low.

The risks from immunosuppression include increased infections and decreased renal function even though the latter does not seem to affect young and healthy patients, who are the target patients for UTx. The long-term potential risk for malignancies such as diabetes mellitus, skin cancer and lymphoma $[20,21]$ typically present after several years of treatment, which would not be required in UTX.

The known risks of pregnancies are the same observed in women with other transplanted organs, such as pre-eclampsia and preterm delivery but also an acceleration of hypertension, new-onset diabetes mellitus and newly arising infection [22]. Besides these, there are unknown risks depending on the fact that the transplanted organ is the uterus, which during pregnancy is expected to function under considerable stress. 
There are psychological risks as well: in living kidney transplantation it has been reported that recipients may experience "shame, anxiety... and guilt over involving another healthy individual in their plight" [23].

Also, psychological risks related to failure of the transplant cannot be excluded.

As regarding the donor, there may be psychological and social benefits deriving from donation. Helping a close relative or a good friend to fulfill a profound desire like pregnancy could make the donor feel valuable and, if the result is the birth of a healthy child, psychological well-being and quality of life may last for many years [19]. The physical risks for a uterus donor are the same related to hysterectomy surgery, even though, as compared to a standard hysterectomy, the duration of the entire surgical procedure here is longer, due to the need to preserve long pedicles of the uterine arteries and veins [24]. In a description of the donor surgery, Brännström et al. reported a 10 to 13 hours operation and a postoperative hospital stay of 6 days, implying additional risks related to anesthesia and other surgical complications [25].

The common complications of simple total abdominal hysterectomy include hemorrhage, transfusion, infection, surgical site, wound site, pelvis, urinary tract, pneumonia, bladder injury, intestinal injury, ureteral injury, and ureterovaginal fistula $[24,25]$.

If the donor is a menopausal woman, she has to take combined oral contraceptives for 90 days before the transplant in order to optimise the uterine vasculature, which involves increased risk of thromboembolic events before and immediately following surgery [26]. 
There may be also psychological risks for the donor. After the transplant, there may be consequences such as depression, anger, disillusionment, and a sense of betrayal, as it has been reported in some kidney donors [23].

Also, there may be consequences already reported in hysterectomized patients, including effects on gender identity and sexuality [27], decrease in sexual satisfaction and increase in sexual dysfunction $[28,29]$.

In 2008, the International Federation of Gynecology and Obstetrics (FIGO) dedicated a report to Uterine transplantation where it was stated that "given the lack of data on safety and the known hazards to live donors, the procedure is considered ethically inappropriate" [30].

In 2013 a prospective observational study of uterus transplantation was initiated, exploring the medical complications and psychosocial wellbeing of the donors for one year after surgery. As regarding medical complications, this study reports one major surgical complication, a ureteric-vaginal fistula, and two self-reported and transient complications (nocturia, meralgia paresthetica). Hospital stays of all donors were 6 days and median sick leave was 56 days (range, 14-132). One year after the intervention, all donors returned to their previous levels of physical health. As regarding psychosocial wellbeing, two donors exceeded 10-point declines in SF-36 summary scores and increased their Hospital Anxiety and Depression Scale (HADS) scores by 6 points during the observation period [31].

Overall, the available data on safety do not indicate a favorable balancing of benefits and risks for the donor, though the autonomous decision of a donor may deserve due respect. Indeed, as compared to lifesaving transplants from living donors, where the urgency of the situation may put the potential donor under 
considerable stress and compromise his/her autonomy [32], in UTx the donor has all the time that she needs to make a weighted and autonomous decision.

The wellbeing of the future child deserves special attention since, as compared to the donor and recipient, who are accepting a certain level of risks in total autonomy throughout the informed consent process, the child is not consenting to the additional risks of being carried in a transplanted uterus.

The risks for the fetus relate primarily to the particular pregnancy that may develop in a transplanted uterus and the potential side effects of immunosuppressive treatment that is needed to avoid rejecting the transplanted uterus.

Unlike other organs, the uterus during pregnancy requires an exceptional vascular plasticity with an increase in blood supply to the uterus from 45 to $750 \mathrm{ml} / \mathrm{min}$, to accommodate a growing fetus [33]. A pregnancy carried in a transplanted uterus may involve increased risks of blood clots and compromised blood supply, which would negatively affect fetal growth and predispose to pregnancy complications including late abortion, preterm birth, pre-eclampsia, fetal growth restriction and placental abruption [35].

The risk with immunosuppressive drugs is that, even in negligible quantities they do cross the placenta and could affect the developing fetus. Their assumption has been found to be associated with an increased risk of miscarriage, prematurity, intrauterine growth retardation and low birthweight, but not of fetal malformation rates $[34,35]$. However, these risks have been reported for certain drugs only, thus regimens can be formulated with a view to minimize gestational harm [36, 37]. A few cases of delivery following UTx have been described by the literature $[38,39]$, and they report an increased risk of preeclampsia and premature birth. However, these complications presented in women with Mayer-Rokitansky-Küster-Hauser 
(MRKH) syndrome, a main cause of AUFI which is characterized by congenital aplasia of the uterus and the upper part (2/3) of the vagina and which is sometimes associated to single kidney function [26]. Therefore, more data are needed to understand whether complications were due to MRKH rather than to UTx.

In the cases described by Brännström and colleagues, children were born respectively at 31 full weeks and 5 days, and 34 full weeks and 6 days, thus they were not severely premature. However, we cannot exclude a higher possibility of severe prematurity on the child, with effects such as cerebral palsy, intellectual disability, visual (retinopathy of prematurity) and hearing impairments, among others [37].

Like any human experimentation placing the fetus at risk, UTx must be extensively considered from an ethical standpoint prior to being accepted as routine practice [39].

\section{Current Research on UTx}

Due to the considerable known and unknown risks of UTx to the recipients, donors and children that may be born thanks to the procedure, the group of experts and other stakeholders that published in 2012 the "Indianapolis Consensus" stated that “(...) clinicians have an obligation toward the patients and society, to closely monitor and register the outcome of the procedure and to define a satisfactory outcome as no less than a live birth. If this is not achieved, the procedure should not be allowed to be instituted as a treatment option" [41].

According to the Indianapolis consensus, before becoming an accepted practice, UTx will have to satisfy the criteria for any surgical innovation: research background, field strength and institutional stability; and satisfy accepted 
bioethical principles (respect for autonomy, beneficence, non-maleficence and justice) and their application $[8,41]$.

Moreover, a defined number of transplants should not be executed worldwide without a successful term delivery, to minimize proceeding in futility using current techniques. This last proposal recalls the criteria of futility, which is usually applied in RCTs either in the protocol or as a request of the Independent Data Monitoring Committee (IDMC) for the conduction of interim analysis [42]. However, the Indianapolis Consensus does not provide exact numbers nor gives other indications in this sense.

It has been suggested that, in order to facilitate the transition from experimental procedure to clinical practice, international teams working on this issue should establish closer collaborations and possibly a multicenter design to also compensate for local variations in surgical techniques, immunosuppression protocols and patient characteristics [43, 44].

Indeed, this is what is being done by International Society of Uterus Transplantation (ISUTx) that met for the first time in Gothenburg, Sweden in January 2016, and whose first congress was held always in Gothenburg, Sweden, on September 2017 with around 140 delegates from all continents [45].

On January 2018, 13 UTx studies were registered on clinicaltrials.gov, the U.S. National Library of Medicine database of privately and publicly funded clinical studies conducted around the world [46] (Table 1).

Of these, 1 study is no longer recruiting (the first UTx trial conducted at the Sahlgrenska University Hospital in Gothenburg, Sweden), 7 studies are actively recruiting, 1 is enrolling participants by invitation only, and 4 are not recruiting yet. 
Nearly all the studies estimate to enroll 10 participants, two of which including 5 donors and 5 recipients, and one study will include only 5 participants.

It is interesting to note that few studies completed the field "Phase" of the study on clinicaltrials.gov, which is typically required for the description of clinical trials. Of these, two studies classified themselves as "Phase 1" and one as "Observational: cohort (Patient registry)", which is the study leaded by Brännström and colleagues. This suggests that transplant researchers are not themselves sure about where should they position the experimental UTx along the continuum between "research" and "practice" [47], and that those that are indicating the "Phase" of the study have an opposite view about their position in the continuum, which here depends much on the expertise of the single transplant team.

Despite the proposal by Brännström et al. to restrict initial studies to the deceased donor concept [44], only 5 studies are planning to limit to transplants from deceased donors, one is planning to include both living and deceased donors and 4 studies will involve living donors.

Studies plan to last from a minimum of 3 years up to 7 years. To be eligible, patients will have to be aged from 18 years up to 40 , or even 45 years (but in this last case embryos must have been produced between the age of 21-39), according to the studies (Table 2).

Studies report from 5 to about 20 inclusion and exclusion criteria including psychological evaluation, stability of the couple and information on the alternatives to family building in one study and a detailed description of exclusion criteria for the donor in one study. Studies also report different primary and secondary outcome measures and different time frames to estimate such measures. 
The most frequent primary outcome measure is the "Number of successful live births after UTx and In Vitro fesrtilisation (IVF)" but also "Transplant success with no clinical, immunological or radiological signs of graft rejection within the first 12 months post-operatively".

Secondary outcomes include the "Rate of pregnancy complications after IVF and uterus transplant", including: hypertension, pre-eclampsia, intrauterine growth restriction, premature rupture of membranes, preterm delivery, intrauterine fetal demise; and the "Rate of neonatal complications" including: birth defects, perinatal infections, low birth weight, neonatal death, neonatal intensive care unit admissions.

UTx complications are followed for 39 up to 41 months after transplantation (in the studies that are indicating the time frame).

Only one study declares the intention to monitor the "Rate of complications following uterine donation", including monitoring for excessive bleeding, infection and bladder dysfunction, and "Impact of uterine donation on donor quality of life" within a time frame of up to 2 years post donation.

The same study intends to assess cost comparison for uterine transplant vs. surrogacy vs adoption also by measuring the impact of uterine transplant on quality of life.

The observational patient registry by Brännström et al. intends to monitor "Development of children after uterus transplantation" for a period of 8 years.

Even though we acknowledge that the information provided on clinicaltrials.gov may be limited as compared to the original study protocols, the picture emerging from that information deserves a few comments. 
1) The declared inclusion and exclusion criteria, primary and secondary outcomes of the different studies vary widely. Also, it is not clear whether the number of participants in studies refers to recipients only or to couples of recipients and donors as specified by two studies. The different study results will probably be comparable, but then it would be useful to know which measures have been taken or will be taken in order to ensure comparability.

2) There is some difficulty in establishing the different phases of such studies, and the difficulty remains even if, instead of the typical 4 RCTs phases, we make reference to the surgery tailored IDEAL classification. By reading the literature, one would have the impression that the primary focus of UTx studies is on safety, thus between stage 2a): Development; and $2 \mathrm{~b}$ ): Exploration. However, the most common primary intended outcome declared here is the "Number of successful live births after uterus transplant", thus an efficacy measure. This is in line with the recommendations in the Indianapolis Consensus, and it is indisputable that the number of livebirths is the most important outcome to be verified, especially if, as always recommended by the Indianapolis Consensus, we need to “(...) minimize proceeding in futility using current techniques". However, the need to study efficacy must not obscure the focus on safety, which is currently a primary concern. It would be useful, in our view, to have on clinicaltrials.gov or other reporting database, a field where researchers can indicate the stage of experimentation for their study, and, for earliest phases, they should report which safety related outcomes they are actively searching for, like a few studies did. 
3) Finally, most UTX studies are planning to transplant the uterus from living donors, but only one study is declaring to follow up the rate of complications following uterine donation (excessive bleeding, infection and bladder dysfunction) and quality of life in donors in the long run.

There may be different reasons why the UTx Centers decide to perform the intervention using uterus retrieved from living donors. The only cases of livebirths following UTx, from the first study up to now, came from transplants from living donors. Even though in deceased uterus donation there would be no surgical risks for the donor, the positive results achieved with living donation may foster a perception of higher probabilities of success using this technique.

However, according to Dani Ejzenberg and colleagues, the viability and potential success of UTx from deceased donors still need to be assessed and would benefit form a better understanding of how to maintain the deceased donor, improve the preservative solution and the perfusion pumps [48].

Finally, there may be difficulties in retrieving uterus from the deceased. Del Priore and colleagues report that in a period of 6 months in New York City (USA) they identified as potential procedures for uterine donation about 150 multiorgan procurements, but uterine donation, which required a specific consent, was accepted in only $6 \%$ of cases. According to the authors the low consent rate could be explained by the fact that the organ in that case would have been used solely for a research purpose. If so, once the procedure became routine practice, then acceptation could also improve as the transplant in that case would be proposed for therapeutic purposes. 
However, non-acceptance may also be due to the symbolic nature of the womb and its reproductive function.

More evidence on the social acceptability of deceased uterus donation in the general population would be useful in order to make a realistic estimate of the number of uterus that may be available from cadaveric donors. Hopefully, the UTx trials that are planning to retrieve uterus from deceased donations will contribute to the study field, even though these objectives do not appear amongst their primary and secondary study outcomes.

Likewise, we hope that all UTx trials that are planning to retrieve uterus from living donors will collect high quality data on the safety of the donor surgery and on the consequences of donation for the donors, even in the long run, but this is not reported amongst primary or secondary outcomes of UTx studies involving living donations.

\section{Conclusions}

Following the first cases of livebirth in 2014 in Sweden, a number of UTx studies is being conducted in different centers worldwide with the aim of assessing safety and efficacy of the procedure for recipients, donors and future children.

Other centers are showing interest for the procedure in Europe, Latin America, Beirut in the Middle East, Asia, and Brisbane in Australia and some centers are receiving surgical training and theoretical sessions by Brännström's equipe in India, Lebanon, Singapore and Argentina [49].

Thus, despite the lack of data on safety, there is optimism among experts that the procedure will not be abandoned and, sooner or later, it will become part of routine clinical practice. 
As for other past transplants, besides available cumulated evidences and expert capacity to find a consensus on best practices, the decision about "routine practice readiness" in UTx will likely depend on social political considerations, including levels of social acceptance of the procedure and pressures form patients and patient groups $[50,51]$.

Transplant teams involved in UTx trials have a role in many ways in shaping the social acceptance of (and willingness to pay for) the procedure.

Improvements in current techniques in non-vital transplants like UTx are shifting societal perspectives and positions on what is considered acceptable surgical risk for such procedures [52], and the promising results achieved in the Swedish trial are also supporting the argument that AUFI is becoming 'treatable'.

By investing energy and funds into UTx studies, doctors are not just responding to women's requests for this option, but they are helping to socially shape that desire and sending the message that becoming a biological mother, and donating one's uterus to make it happen, is worth the risk [53].

In an interview, Brannstrom reports that, since the first successful uterus transplants resulted in a livebirth, he continues to receive approximately 50 emails a day from women requesting the surgery [54].

Likewise, a survey conducted on 40 women with AUFI indicated an overwhelming preference of women for UTx over surrogacy and adoption $(n=39,97.5 \%)$ even knowing that the latter 2 options are safer for them and the fact that the graft could fail prior to conception. All women also supported that UTx should take place as an option [55].

However, in the enthusiastic climate that accompanies such extraordinary success, we foresee two main concerns. 
One, already highlighted and partly confirmed by our review of current studies on clinicaltrials.gov, is the risk that the competition to see which research team will achieve the highest success rate may negatively impact the science behind it [56]. Recommendations to avoid this risk come from the World Medical Association Statement on Human Organ Donation and Transplantation stating that: "the WMA considers that, although many transplantation procedures have become standard medical care for a range of medical conditions, others are experimental and/or morally controversial and require further research, safeguards, guidelines and public debate. Experimental procedures require protocols, including ethics review, that are different and more rigorous than those for standard medical procedures" [57].

The other concern relates to the potential consequences for all potential uterus donors.

If UTx became part of accepted routine practice, the procedure would become more easily accessible for women with AUFI, and potential recipients and donors, as well as their intimate relations, would necessarily be confronted with this new extraordinary possibility.

However, an ethical concern is that, in close family personal relationships, potential donors may feel under familial or social pressure to take risks and act against their own interests for the benefits of others close to them [58].

It may be difficult to discern whether a prospective donor is under "undue pressure" from the rest of the family or from her conscience, including nonrational feelings of responsibility and guilt.

Early research on kidney living donations report that many of the prospective donors interviewed by physicians said they felt that they had "no choice," that in 
most cases they were not enthusiastic about donation, but felt just unable to refuse [48].

In case UTx became routine practice, The Indianapolis Consensus recommends to keep pursuing additional research objectives:

- $\quad$ keep studying pregnancies following UTx in different animal models (to search for unanticipated consequences);

- $\quad$ regularly assess the opinion of women with AUFI regarding UTx;

- $\quad$ regularly assess potential recipients using 'borrowed' psychological tools from transplant centers, adoption agencies and Assisted Reproduction Technologies (ART) centers (iv) conduct careful ethical reflection, assessment and approval.

We would add to this "regularly assess the opinion of living donors and potential living donors".

If in TS, besides scientific evidence, the decision on "routine practice readiness" of a new procedure also involves a political and social dimension, it would be time to discuss how to embed these dimensions in more structured and formal ways, with a view to representing the point of view of all involved parties.

UTx studies that are being carried worldwide could widely contribute to this enterprise by collecting more data on donors' opinions before and after surgery thus improving besides the "field strength", also the process of approval of new procedures in TS more in general.

\section{Declarations}

Ethics approval and consent to participate: Not applicable Consent for publication: Not applicable 
Availability of data and material: Not applicable (the paper does not present original data but some data are reused from clinicaltrials.gov:

https://clinicaltrials.gov/ct2/results?term=uterine+transplantation\&pg=1)

Competing interests: The authors declare that they have no competing interests.

Funding: None

Authors' contributions

SG conceived the paper and prepared the main text

$\mathrm{CP}$ conceived the paper and revised the text

Acknowledgements

Not applicable 


\section{References}

1. Fox RC. Experiment Perilous: Physicians and Patients Facing the Unknown. Glencoe, IL: Free Press; 1959.

2. Fox RC, Swazey JP. The courage to fail (Chapter 3). Oxford University Press, Oxford; 2001.

3. Evans RW. Organ transplantation costs, insurance coverage, and reimbursement. Clin Transpl. 1990:343-55.

4. European Parliament and the Council. Directive 2001/83/EC of the European Parliament and the Council of 6 November 2001 on the Community code relating to medicinal products for human use. Official Journal of the European Communities L 311(4)28.11.2001. Article 8, point 3, comma i.

5. U.S. Department of Health and Human Services. U.S. Food and Drug Administration. New Drug Application (NDA). Last Update: 03/29/2016 https://www.fda.gov/Drugs/DevelopmentApprovalProcess/HowDrugsare DevelopedandApproved/ApprovalApplications/NewDrugApplicationNDA/ default.htm (Accessed on February 5, 2018).

6. The IDEAL Collaboration. http://www.ideal-collaboration.net/framework/

7. Schlich $\mathrm{T}$. The origins of organ transplantation. The Lancet. 2011;378(9800):1372-3.

8. Moore FD. Ethical problems special to surgery: surgical teaching, surgical innovation, and the surgeon in managed care. Arch of Surg. 2000; 135:1416.

9. Morgenstern L. Innovative Surgery's Dilemma. Surg Innov. 2006;13:73.

10. McCulloch P, Altman DG, Campbell WB, Flum DR, Glasziou P, Marshall JC, et al. No surgical innovation without Evaluation: the IDEAL Recommendations. The Lancet 2009;374 (9695):1105-1112.

11. Editorial. Surgical Research: the Reality and the IDEAL. The Lancet 2009;374(9695):1037.

12. Fox RC, Swazey JP. (1992) Spare parts. New York, Oxford University Press. P.9.

13. Petrini C, Gainotti S, Morresi A. Ethical issues in uterine transplantation: psychological implications and informed consent. Transplantation Proceedings 2017;49(4):707-710. DOI:

https://doi.org/10.1016/j.transproceed.2017.02.013

14. Erman Akar M, Ozkan O, Aydinuraz B, et al. Clinical pregnancy after uterus transplantation. Fertil Steril 2013;100:1358-1363.

15. Akouri R, Maalouf G, Abboud J, et al. Uterus transplantation: an update and the Middle East perspective. Middle East Fertility Society Journal 2017; 22(3):163-169. https://doi.org/10.1016/j.mefs.2017.04.003

16. Brännström $M$, Johannesson $L$, Dahm-Kähler $P$, Enskog $A$, Mölne J, Kvarnström N, et al. First clinical uterus transplantation trial: a six-month report. Fertil Steril 2014;101:1228-1236. doi:

10.1016/j.fertnstert.2014.02.024. 
17. Johannesson L, Kvarnström N, Mölne J, Dahm-Kähler P, Enskog A, DiazGarcia C,et al. Uterus transplantation trial: 1-year outcome. Fertil Steril 2015;103:199-204. doi: 10.1016/j.fertnstert.2014.09.024

18. Sifferlin A. Exclusive: First U.S. baby born after a uterus transplant. Time Dec 1, 2017 http://time.com/5044565/exclusive-first-u-s-baby-born-aftera-uterus-transplant/ (Accessed on February 5, 2018).

19. Olausson M, Johannesson L, Brattgard D, Diaz-Garcia C, Lundmark C, Groth $\mathrm{K}$, et al. Ethics of uterus transplantation with live donors. Fertil Steril. 2014;102(1):40-43. doi: 10.1016/j.fertnstert.2014.03.048.

20. Pham PT, Pham PC, Lipshutz GS, Wilkinson AH. New onset diabetes mellitus after solid organ transplantation. Endocrinology and Metabolism Clinics of North America. 2007;36(4);873-890.

21. Srisawat N, Avihingsanon A, Praditpornsilpa K, Jiamjarasrangsi W, EiamOng $S$, Avihingsanon Y. A prevalence of post transplantation cancers compared with cancers in people with human immunodeficiency virus/acquired immunodeficiency syndrome after highly active antiretroviral therapy. Transplant Proc. 2008 Oct;40(8):2677-9.

22. Blume $C$, Pischke $S$, von Versen-Höynck F, Günter HH, Gross MM. Pregnancies in liver and kidney transplant recipients: a review of the current literature and recommendation. Best Pract Res Clin Obstet Gynecol. 2014;28(8):1123-36.

23. Sajjad I, Baines LS, Salifu M, Jindal RM. The dynamics of recipient-donor relationships in living kidney transplantation. Am J Kidney Dis. 2007;50(5):834-54.

24. Kisu I, Mihara M, Banno K. Risks for donors in uterus transplantation. Reproductive Sciences. 2013;20(12):1406-1415.

25. Brännström M, Johannesson L, Dahm-Kähler P, Ensko, A, Mölne J, Kvarnström N., et al. First clinical uterus transplantation trial: a six-month report. Fertil Steril. 2014;101:1228-36.

26. Farrell RM, Falcone T. Uterine transplant: new medical and ethical considerations. The Lancet. 2015;385(9968):581-82.

27. Vomvolaki, E., Kalmantis, K., Kioses, E., Antsaklis, A. The effect of hysterectomy on sexuality and psychological changes. Eur J Contracept Reprod Health Care. 2016;11:23.

28. Sozeri-Varma G, et al. The effect of hysterectomy and/or oophorectomy on sexual satisfaction. Climacteric: J Int Menopause Soc. 2011; 14: 275.

29. Carter J, Sonoda Y, Baser RE, Raviv L, Chi DS, Barakat RR. et al. A 2-year prospective study assessing the emotional, sexual, and quality of life concerns of women undergoing radical trachelectomy versus radical hysterectomy for treatment of early-stage cervical cancer. Gynecol Oncol. 2010;119: 358.

30. Milliez, J. Uterine Transplantation FIGO Committee for the Ethical Aspects of Human Reproduction and Women's Health. Int J Gynaecol Obst. 2008;106:270. (Point 4.) 
31. Kvarnström N, Järvholm S, Johannesson L, Dahm-Kähler $P$, Olausson $M$, Brännström $M$.

Live donors of the initial observational study of uterus transplantationpsychological and medical follow-up until 1 year after surgery in the 9 cases. Transplantation. 2017;101(3):664-670. doi: 10.1097/TP.0000000000001567.

32. Singer PA, Siegler M, Whitington PF, Lantos JD, Emond JC, Thistlethwaite $J R$, et al. Ethics of liver transplantation with living donors. N Engl J Med 1989; 321:620-622. DOI: 10.1056/NEJM198908313210919

33. Brosens I, Ghaem-Magham S, Pijnenborg R. Uterus transplantation in the human: a complex surgical, medical and ethical challenge. Hum Reprod. 2013;28(2):292-93.

34. Brosens I, Pijnenborg R, Vercruysse L, Romero R. The 'Great Obstetrical Syndromes' are associated with disorders of deep placentation. Am J Obstet Gynecol. 2011;204:193-201.

35. Tendron A, Gouyon J-B, Decramer S. In utero exposure to immunosuppressive drugs: experimental and clinical studies. Pediatr Nephrol. 2002(17):121-30.

36. Lefkowitz A, Edwards M, Balayla J. Ethical considerations in the era of the uterine transplant: an update of the Montreal criteria for the ethical feasibility of uterine transplantation. Fertil Steril. 2013;100(4):924-6. doi: 10.1016/j.fertnstert.2013.05.026.

37. Lefkowitz A, Edwards M, Balayla J. The Montreal Criteria for the Ethical Feasibility of Uterine Transplantation. Transpl Int. 2012 Apr;25(4):439-47. doi: 10.1111/j.1432-2277.2012.01438.x.

38. Brännström, M., Johannesson, L., Bokström, H., Kvarnström, N., Mölne, J., Dahm-Kähler, P., et al. (2015). Livebirth after uterus transplantation. Lancet;385(9968):607-16.

39. Brännström M, Bokström H, Dahm-Kähler P, Diaz-Garcia C, Ekberg J, Enskog $A$, et al. One uterus bridging three generations: first live birth after mother-to-daughter uterus transplantation. Fertil Steril. 2016;106(2):2616.

40. Daar J, Klipstein S. Refocusing the ethical choices in womb transplantation. J Law Biosciences. 2016;383.388.

41. Del Priore G, Saso S, Meslin EM, Tzakis A, Brännström M, Clarke A, et al. Uterine transplantation--a real possibility? The Indianapolis consensus. Hum Reprod. 2013 Feb;28(2):288-91. doi: 10.1093/humrep/des406.

42. Jitlal M, Khan I, Lee SM, Hackshaw A. Stopping clinical trials early for futility: retrospective analysis of several randomized clinical studies. $\mathrm{Br} \mathrm{J}$ Cancer. 2012;107(6):910-917.

43. Saso S, Ghaem-Maghami S, Louis LS, Ungar L, Del Priore G, Smith JR. Uterine transplantation: what else needs to be done before it can become a reality? J Obstetrics Gynaecology. 2013; 33(3): 232-238.

44. Johannesson L, Dahm-Kähler P, Eklind S, Brännström $M$. The future of human uterus transplantation. Women's Health. 2014; 10(4):455-467. 
45. International Society for Uterus Transplantation.

http://www.isutx.org/about/ (Accessed on February 5, 2018).

46. Clinicaltrials.gov. Uterine transplantation. Research Results. Retrieved March 23, 2017, from:

https://clinicaltrials.gov/ct2/results?term=uterine+transplantation\&pg=1

47. Singer PA, Siegler M, Lantos JD, Emond JC, Whitington PF, Thistlethwaite $J R$, et al. The ethical assessment of innovative therapies: liver transplantation using living donors. Theor Med. 1990 Jun;11(2):87-94.

48. Dani Ejzenberg I, Baratelli Carelli Mendes LR, Bertocco de Paiva Haddad L, Chada Baracat E, Carneiro D'Albuquerque LA, Andraus W. Uterine Transplantation: a systematic review. Clinics (Sao Paulo). 2016 Nov; 71(11): 679-683.

49. International Society for Uterus Transplantation. Founding members. http://www.isutx.org/founding-members/ (Accessed on February 5, 2018).

50. Fox RC. A sociological perspective on organ transplantation and hemodialysis. Ann N Y Acad Sci. 1970 Jan 21;169(2):406-28.

51. Evans RW. Insurance coverage of heart transplantation in the United States: The dilemma, the debate and the definitive decision that ultimately determined the future of transplantation. J Heart Lung Transplant. 2017 Dec;36(12):1294-1298. doi: 10.1016/j.healun.2017.10.004.

52. Haberal M, Dalgic A. New concepts in organ transplantation. Transpl Proc 2004;36: 1219.

53. Rudavsky S, Star I. Uterine transplants: a new frontier in science. Center for Genetics and Society. 2011. Retrieved on march 30, 2017, at: http://www.geneticsandsociety.org/article.php?id=6002

54. Pondrom S. US to Offer Uterus Transplants. The AJT Report 2016;16:2. DOI: 10.1111/ajt.13718

55. Saso S, Clarke A, Bracewell-Milnes T, Saso A, Al-Memar M, Thum MY, et al. Psychological issues associated with absolute uterine factor infertility and attitudes of patients toward uterine transplantation. Prog Transplant. 2016;26(1):28-39.

56. Caplan AL. Ensuring the future of uterine transplantation. Fertil Steril 2013;99(3):682-683. DOI:

https://doi.org/10.1016/j.fertnstert.2012.11.010

57. World Medical Association. World Medical Association Statement on Human Organ Donation and Transplantation. Adopted by the 52nd WMA General Assembly in Edinburgh, Scotland during October 2000 and revised by the WMA General Assembly, Pilanesberg, South Africa, October 2006. Retrieved on march 30 at: http://www.wma.net/en/30publications/10policies/wma/index.html.

58. Dickens, B.M. (2016). Legal and ethical issues of uterus transplantation. Int J Gynaecol Obstet. 133(1):125-8. 